\title{
Towards Adaptive Generation of Mathematical Exercises
}

\author{
Imane Lmati \\ Analysis, Modeling and \\ Simulation Laboratory \\ University Hassan II, Morocco
}

\author{
El habib Benlahmar \\ Laboratory of Information \\ Technology and Modeling, \\ University Hassan II, Morocco
}

\author{
Naceur Achtaich \\ Analysis, Modeling and \\ Simulation Laboratory, \\ University Hassan II, Morocco
}

\begin{abstract}
This article presents a new approach for teachers to adaptive generation of self-evaluation exercises according to the profile of students. It allows the creation of different exercises (Direct exercises, Complex exercise...). Each type mathematical exercise helps to give an evaluation in various concepts. The author approach consists of three phases: pre-test phase, filtering phase and evaluation phase. The first phase is devoted to determining the level of mastery of the learner about basic notions by updating the ontology profile using the rating obtained for each notion (concept). For the second phase, filter concepts is carried out by keeping the well mastered concepts. For the last phase, it is for the generation of extended exercise model (high difficulty) based on all the possible combination of concepts mastered. The ability to generate several exercises from the same model allows the learner to evaluate several times on the same concepts without the teacher having to repeatedly define many exercises.
\end{abstract}

\section{Keywords}

Adaptive generation of exercises; authoring tool; Selfevaluation; automatic evaluation.

\section{INTRODUCTION}

Student assessment is a very important issue in educational community [1]. The main goal of an evaluation process is to collect information about student abilities, determine the level of the student's knowledge and to identify the skills that students can do and those that they can't. This acts a feedback for making decisions about the learning environment, especially if we plan to use the evaluation process as a diagnostic tool to matching the content of online course to individual students. The evaluation strategy of students differs widely throughout the world. Different methods of knowledge evaluations are in use, such as in class presentations, writing essays, projects... etc. However, the most common "tool" that is used to measure knowledge is the test tool.

For adaptive tests. It is designed to estimate the level of learner skills. There are many adaptive e-assessment systems and tools that implement adaptive testing techniques such as: $[2,3,4,5,6$, and 7]. An adaptive test represents an advantage for improve the skills and to better diagnose the level of learners. They can be used to generate adaptive learning contained (courses, pedagogical scenario, exercise ...)

In this work, we arrived to exploit it by generating educational content as mathematical exercises. The test is performed as direct exercises useful to power profile model. This allowed us to generate more advanced exercises adequate to the needs of the learner.
This proposal refers to two theoretical models. The first is represented by an ontology of profile [8] powered by a

pre-test to determine the real level of the learner. The second model is ontology for mathematics content $[9,10]$ extracted from the pedagogical courses. The latter is the basis for automatic generation of mathematics exercises. The following section focuses light on the approach specially knowledge model necessary for the implementation of the approach, after we present the process for the generation of pedagogical contained in particular the generation of mathematical exercises based tests. Finally, we perform an evaluation of the methodology developed based on a corpus of mathematical courses of secondary school.

\section{KNOWLEDGE MODEL}

In this section, we describe the knowledge models exploited in the generation process model exercise adaptive, based on the E-Orientation ontology [8] on the one hand and ontology extended Math-Bridge [9].

\subsection{Extended Math-Bridge ontology}

According to [11], mathematics texts (article, educational support ...) consist of entities or specific markers we call "math tags" labeled according to specific forms written by authors. The most common tags are "Theorem, Lemmas, and Definition...." The Math-Bridge project allows modeling all of these labels into a core of ontology

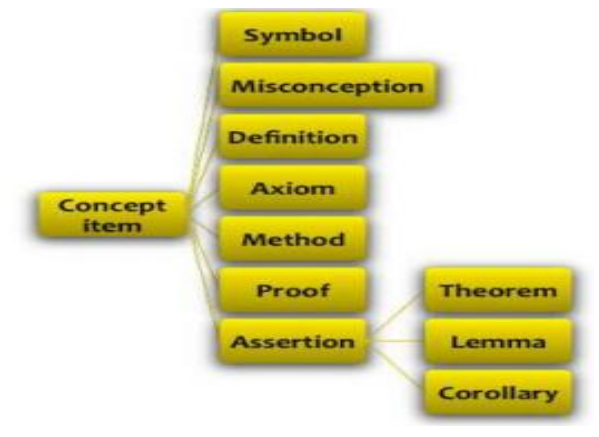

Fig 1: Math-Bridge ontology

The model of the ontology can be extended by other sub concepts to facilitate the navigation and search of knowledge. Each pedagogical objects PO structured in three parts: Variable (parameter used in the PO), Prerequisites (constraint) and output (result of PO). For each variable, we define the name, type, and its domain. The pre-requisites and outputs are separated into logical and textual part to facilitate the extraction of knowledge. Each pre-requisites / output is divided into pre-argument, post-argument and predicate argument. 


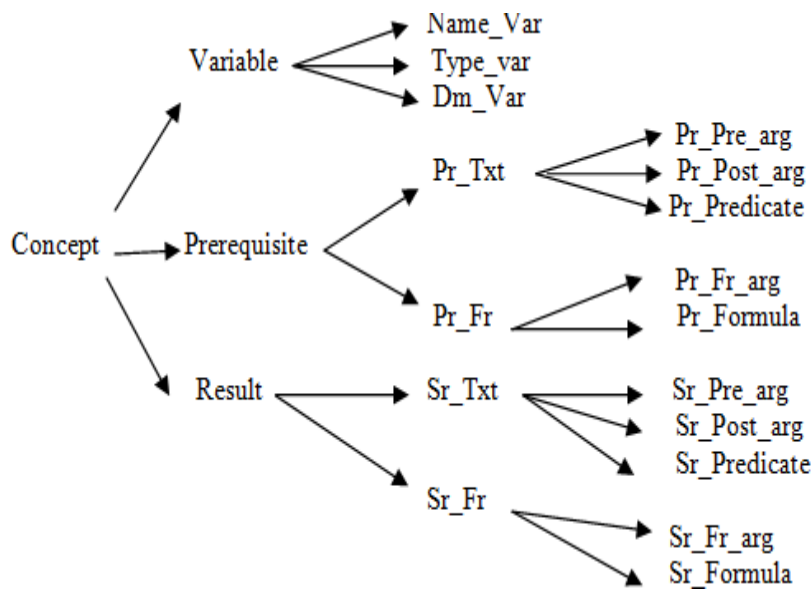

Fig 2: Extension of educational ontology

\subsection{PROFILE ONTOLOGY}

The E-oriented ontology defines the learning profile with different characteristics, using concepts and relationships between various concepts. It describes a learner in four not mastered during his studies. Alternatively, one can annotate each didactic concept DC acquired by a note. aspects as abstract concepts in the ontology. From the ontology profile. We can define the mathematical concepts

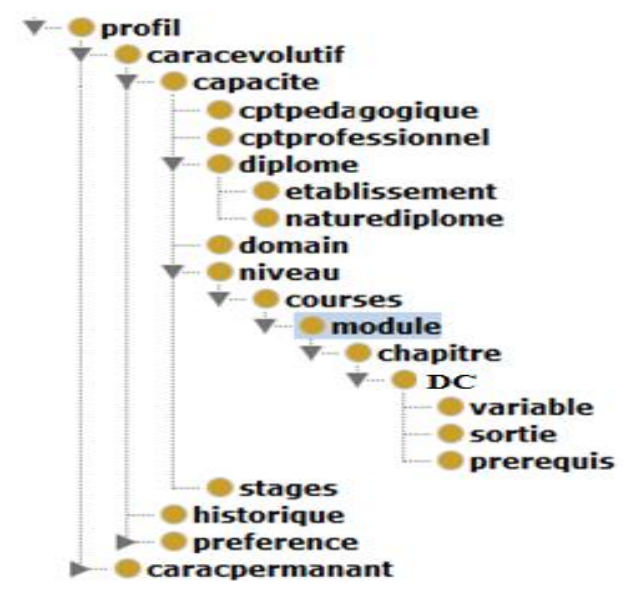

Fig 3: E-oriented ontology

\section{PROCESS ADAPTATIVE ASSESSMENT}

Figure (4) presents the assessment process. It consists of three phases: pre-test phase, filtering phase and evaluation phase.
Let $(\mathrm{Ci})$ a pedagogical concept. ( $\mathrm{Si}$ ) a proposal of concepts linked by semantic relations headed by the concept $\mathrm{Ci}$ and

$(\mathrm{Nb})$ the number of chained concepts for each solution $(\mathrm{Si})$. 


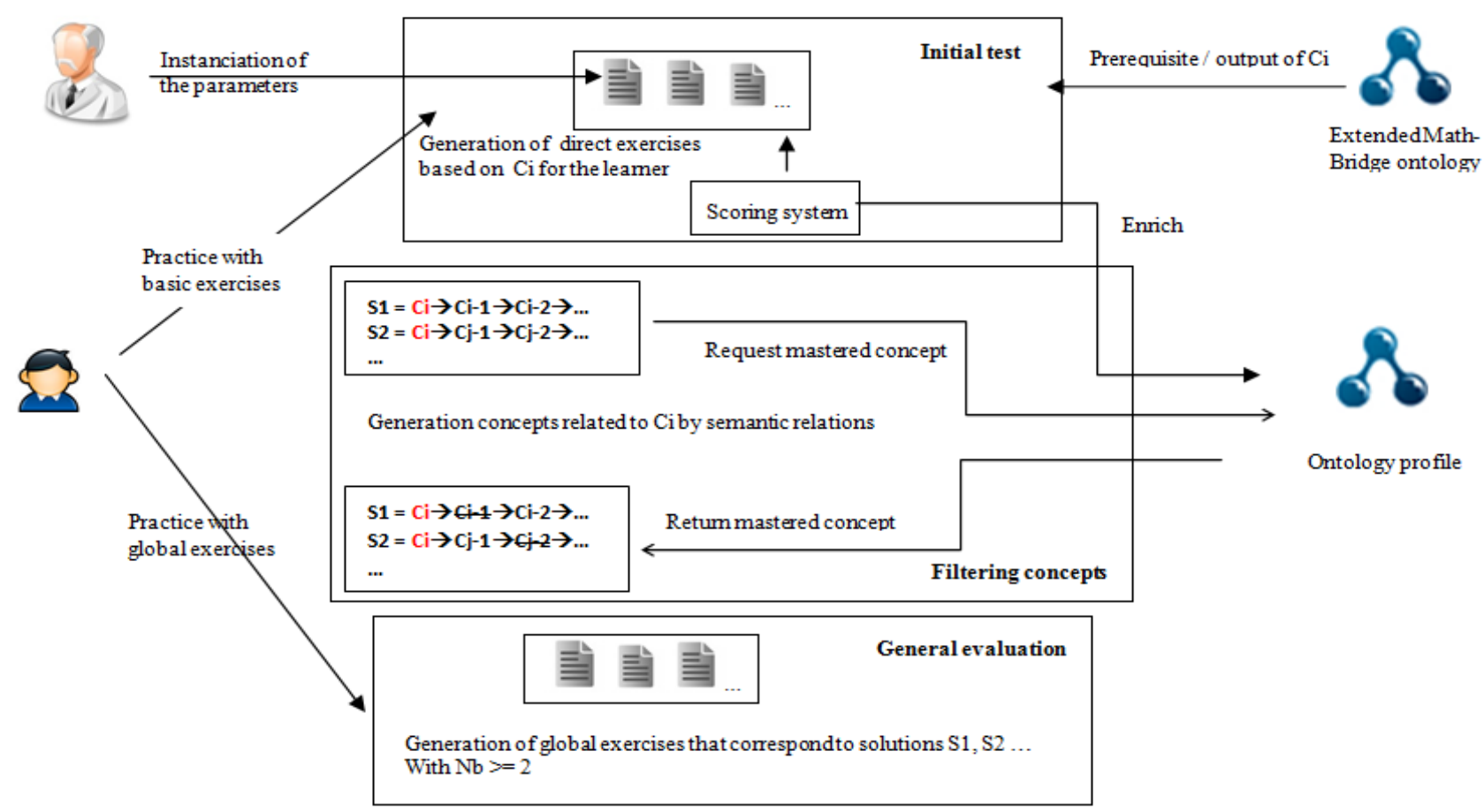

Fig 4: Process adaptive assessment

In the process, the author can manually set the exercises to give an infinite instance. In parallel, the learner is assessed at two levels: the initial test and the general evaluation.

\section{Phase Pre-Test by level of study:}

The first phase is devoted to determining the level of mastery of basic concepts by the learner for a given level of study by updating the ontology profile using the rating obtained for each concept. This phase allows the generation of direct exercises as simple questions which request the result of the concept using its pre-requisites posed (Or requested) in statement of exercise. In this phase the teacher may propose parameter instances to the mathematical model exercise respecting the constraint data in the statement.

\section{Filtering phase of process adaptive assessment:}

Filtering is based on four steps:

1. Designing a basic concepts linked by semantic relations headed by the concept $\mathrm{Ci}$ (Choose from the learner) for the generation of exercises of higher degree of difficulty.

2. Filtering concepts: this step is in relation to the profile ontology which gives the level of mastery of each concept found in the step (1). The filter eliminates concepts not mastered by the learner (score (Ci) $\leq$ threshold).

3. Generation of global exercises corresponding to the solution of step 1 and 2 (S1, S2 ..).

4. Back to the pre-test phase if the number of concepts of each solution $(\mathrm{S} 1, \mathrm{~S} 2 \ldots)$ found is less than two ( $\mathrm{Si}=$ $\mathrm{Cj} \ldots)$.
Post-Test by level of mastery:

This phase is reserved to the generation of adaptive extended model exercise (High difficulty) based on all the combinations of concepts mastered linked by Indirect semantic relations).

\section{CREATION OF ADAPTATIF MODEL EXERCISE}

The creation of the exercise depends on the level of study and mastery of the concept acquired by leaner. The latter is evaluated according to two types of tests:

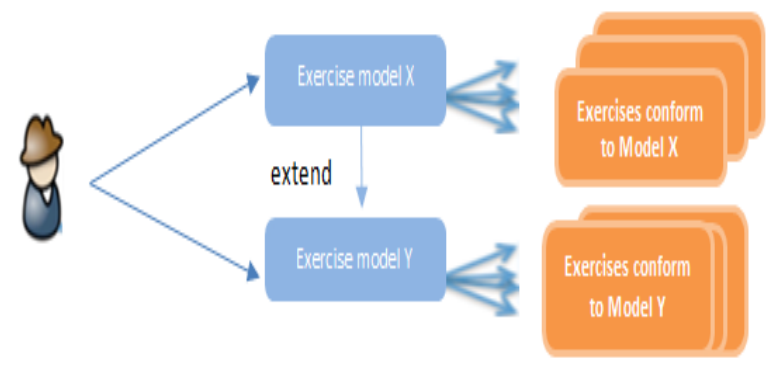

Fig 5: Types of assessment test

\subsection{Model X: Direct exercise}

The type $\mathrm{X}$ of exercise model is created to generate direct exercises instances linked to each educational concept of ontology. This model is generated during the pre-test phase. For the statement, the parameters are manually given by the teacher. The prerequisites and outputs are given as a question. 
Model exercise

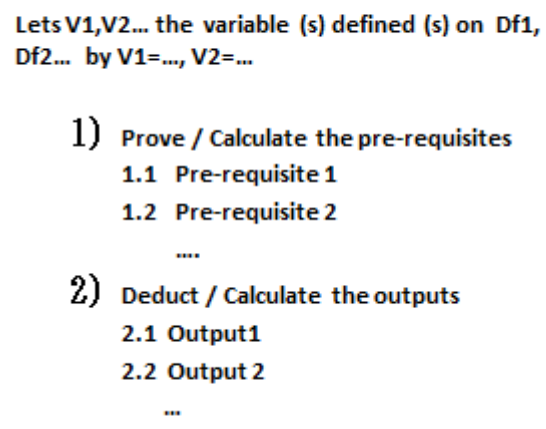

2) Deduct / Calculate the outputs

Exercise Instance (C1)

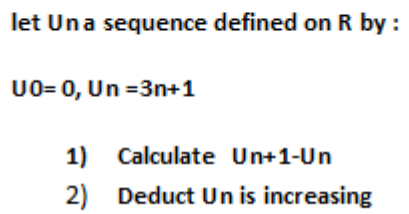

1) Calculate Un+1-Un

2) Deduct $U n$ is increasing

Fig 6: Process for the generation of model $\mathrm{X}$

Example:

Let $\mathrm{C} 1$ a pedagogical concept: «A reel sequence $\mathbf{U n}$ is increasing if and only if for every $n, U n+1 \geq U n$ ».

The statement of the exercise contains input parameter

$(\mathrm{U} 0=0, \mathrm{Un}=3 \mathrm{n}+1)$ and the domain definition (the set $\mathrm{R})$. For the first question, we request to calculate the pre-requisites of the definition of the monotony of reel sequence (Un+1-Un).

For the output, we ask to deduce the result of the concept (C1) which is: "Un is increasing".

\subsection{Model Y: Complex exercise}

The exercise model $\mathrm{Y}$ is an extended model $\mathrm{X}$. This model is generated during the phase of the general evaluation.

This model can process several educational concepts at once to provide some difficulty for the exercise based on the semantic relationship between the concepts selected from the mathematical ontology.

Example:

Let's C1, C2 pedagogical concepts extracts from a mathematical course of the terminal.

To select instances of extended model, one must choose the entered values (parameter) suitable for constraints of the

\begin{tabular}{|c|c|c|}
\hline \multicolumn{3}{|c|}{ Extended model } \\
\hline \multicolumn{3}{|c|}{$\begin{array}{l}\text { Lets } \mathrm{V} 1, \mathrm{~V} 2 \ldots \text { the variable }(\mathrm{s}) \text { defined (s) on Df1, Df2 ... } \\
\text { by } \mathrm{V} 1=\ldots, \mathrm{V} 2=\ldots\end{array}$} \\
\hline \multirow[t]{4}{*}{ 1) } & Prov & ve/Calculate/deduct the prerequisites \\
\hline & 1.1 & Prerequisite \\
\hline & 1.2 & Prerquisite \\
\hline & & $\ldots$. \\
\hline \multirow[t]{3}{*}{ 2) } & Prov & ve/Calculate/deduct the outputs \\
\hline & 2.1 & Output 1 \\
\hline & 2.2 & Output 2 \\
\hline
\end{tabular}

three concepts initially selected. Also, the concepts should also be well mastered by the learner with:

Score $(\mathrm{C} 1)$, Score $(\mathrm{C} 2)$ et $\operatorname{Score}(\mathrm{C} 3) \geq$ Threshold.

C1: A reel sequence $U n$ is increasing if and only if for every $n, U n+1 \geq U n$

C2: If a sequence is increasing and bounded then it converges

\section{C3 : If Un converges then $\operatorname{Lim}$ un+1 $-\mathrm{Un}=\mathbf{0}$}

Table 1. Extraction of the attributes of pedagogical concepts

\begin{tabular}{|c|l|l|}
\hline $\begin{array}{l}\text { Pedagogical } \\
\text { concept }\end{array}$ & Prerequisites & Output \\
\hline $\mathrm{C} 1$ & $\begin{array}{l}\text { for every } \mathrm{n}, \mathrm{Un}+1 \geq \\
\text { Un }\end{array}$ & Un is increasing \\
\hline $\mathrm{C} 2$ & $\begin{array}{l}\text { A sequence is } \\
\text { increasing and bounded }\end{array}$ & it converges \\
\hline $\mathrm{C} 3$ & Un converges & $\begin{array}{l}\text { Lim un }+1- \\
\text { Un }=0\end{array}$ \\
\hline
\end{tabular}

The concepts $\mathrm{C} 1, \mathrm{C} 2$ are connected by a low precedence relation [12]. By against $\mathrm{C} 2$ and $\mathrm{C} 3$ are related by a strong relationship precedence. Since we have Output $(\mathrm{C} 1) \subseteq$ Prerequisite $(\mathrm{C} 2)$ and Output $(\mathrm{C} 2)=$ Prerequisite $(\mathrm{C} 3)$.

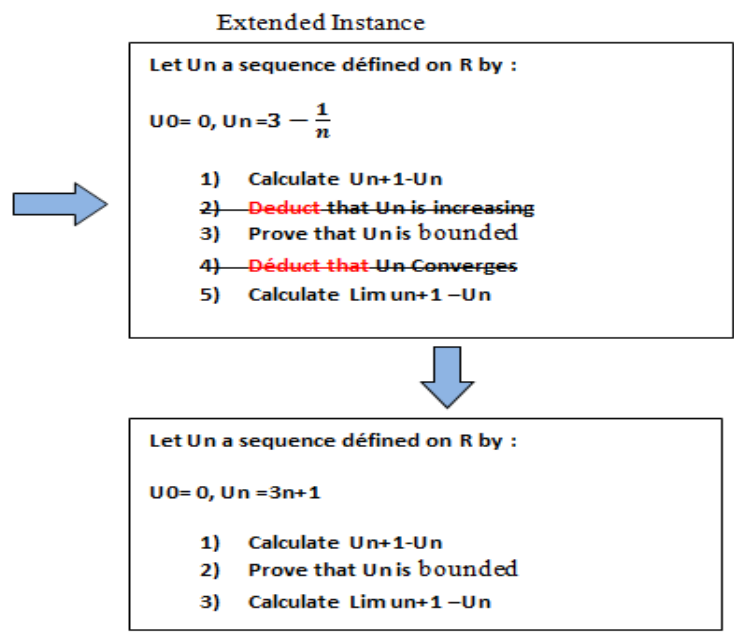

Fig 7: Process for generation of model $Y$ 


\subsubsection{Process}

To generate a model $\mathrm{Y}$ with high difficulty, we used the same approach of model $\mathrm{X}$ for each concept used respecting their orders in high / low precedence relationship $(\mathrm{C} 1 \rightarrow \mathrm{C} 2 \rightarrow \mathrm{C} 3)$.

\section{Extended Instance:}

The first Question involves the pre-requisites $\mathrm{C} 1$. The second question is a deduction result of $\mathrm{C} 1$. The third question involves the pre-requisites of $\mathrm{C} 2$ and since one of the prerequisites is already requested (Question 2: Un is increasing), we just asked the second pre-requisites is "Un is bounded ". The fourth is a deduction of the result $\mathrm{C} 2$. Fifth involves the prerequisite of $\mathrm{C} 3$ and since it is already requested (Un converges) we deduct its output.

\section{Final instance:}

To extract the final model, we try to eliminate anything that is deducted from the statement for extended instance, keeping the question of calculation or demonstration.

\subsubsection{Patterns for the generation of exercise statement}

Based on the last idea, we extract three patterns for the following verbs: Calculate/ Prove/ deduct used in the questions of the exercise.

Prove:

The term "prove" can be used in the questions of exercise to ask the textual or logical prerequisite.

\section{Calculate:}

If one of the attributes of concepts contains a variable (simple, complex) quantified by mathematical operators $\left(\sum, \pm \ldots\right)$,followed by an equal or inequality operator $\quad(=$, $\geq, \leq \ldots$ ), we transfer all variable in one side and we request to calculate the remaining value of the second side of equality or inequality (question 1 of figure 7).

\section{Deduct:}

This Pattern allows asking the textual or logical outputs of each concept used in the exercise (question 4 in Figure 7).

\section{EVALUATION}

We applied the approach to a set of three type of profile for students of secondary education, to generate mathematical exercises with different degree depending on the number of used concept (one, two or three concepts). As starting point, we have chosen the pedagogical concept $\mathrm{C} 1$ which represents the definition of continuity of digital function.

Definition: Let $\mathrm{f}$ a function defined on an interval I containing the real a. So:

- The function $\mathrm{f}$ is continuous at $\mathrm{a}$ if $\lim \mathrm{x} \rightarrow \mathrm{a} f(\mathrm{x})=$ f (a)

- The function $\mathrm{f}$ is continuous on $\mathrm{I}$ if it is continuous at each point I.

All generated concept are linked by strong / low precedence relationship headed by the concept $\mathrm{c} 1$, such as the concepts mastered by the learner based on obtained score (ontology profile).

The concept used are taken from a corpus of mathematical analysis courses of terminal as « $\mathrm{C} 2$ : Intermediate value theorem », «C3: theorem Bijection of numerical functions $»$... Similarly, we try to find other semantic relationship for concept found $\mathrm{C} 2, \mathrm{C} 3$..

For each type of profile and for each degree of difficulty of exercises generated, we evaluate the approach, by: the recall and precision

$$
\text { Precision }=\frac{\text { Number of exercises correctly generated }}{\text { Number } \text { of generated exercises }} \quad \text { Recall }=\frac{\text { Number of exercises correctly generated }}{\text { Number of exercises generated by expert }}
$$

Table 2. Results of adaptive generation of mathematical exercises

\begin{tabular}{|l|c|c|c|c|c|c|}
\hline \multicolumn{1}{|c|}{ Type of profile } & \multicolumn{2}{c|}{ Profile 1 } & \multicolumn{2}{c|}{ Profile 2 } & \multicolumn{2}{c|}{ Profile 3 } \\
\hline $\begin{array}{c}\text { Degree of difficulty of } \\
\text { exercise (number of } \\
\text { concept) }\end{array}$ & Precision & Recall & Precision & Recall & Precision & Recall \\
\hline Low (one conept) & 0,88 & 1,00 & 0,95 & 0,82 & 0,92 & 1,00 \\
\hline Medium (two cocnept) & 0,85 & 0,97 & 0,94 & 1,00 & 0,97 & 0,91 \\
\hline High (tree concept) & 0,33 & 0,03 & 0,25 & 0,03 & 0,33 & 0,03 \\
\hline
\end{tabular}

For direct exercises (number concept equal to 1), we obtained quite a number of instances (converges to the number of exercises generated by expert) based on a manual parameterization (input values for the exercise). By against, for the case of two or three concept, evaluation is pretty good for the three types of profile since it must be based on the same input values for two or three concept used and we also must respect the concept mastered by learner based on the obtained score (profile ontology).

\section{CONCLUSION}

With the advancement of information and communications technology for education, it is increasingly frequently happens that learners work independently with educational content online. To support them in this learning, we want to offer to the author (generally a teacher) the ability to easily create exercises self-assessment adaptive of different difficulty for the learner. Different generators exercises already exists but none has all properties that we hope: Exercise is different from one time to another; the author is master of parametrage to ensure the reliability exercises; the exercise generator is used in many grade levels; a scoring module is used to provide real score of the current level of the learner; building exercise does not take an excessive amount of time for the author; creating exercises do not require special technical skills. We hope also, in future works to: 
- automate the generation of patterns used in the approach to facilitate the creation of the statement exercises

- Develop a first prototype of the approach.

\section{REFERENCES}

[1] Al-Rajhi, L., Reda S., and Shehab G. , 2014, Personalized Intelligent Assessment Model for Measuring Initial Students Abilities. Proceedings of the 2014 Workshop on Interaction Design in Educational Environments. ACM.

[2] Conejo, R., Guzmán, E., Millán, E., Trella, M., Luis, J. and Ríos, A. 2004. SIETTE: A Web-Based Tool for Adaptive Testing. In International Journal of Artificial Intelligence in Education 14, 1 -33. IOS Press.

[3] Machuca. E., Conejo. R., Guzman. E., and Libbrecht. P. 2005. Integrated Adaptive Assessment Tool. The LeActiveMath Consortium.

[4] Tzanavari, A., Retalis, S. and Pastellis, P. 2004. Giving More Adaptation Flexibility to Authors of Adaptive Assessments. In Adaptive Hypermedia and Adaptive Web-Based Systems (pp. 340-343). Springer Berlin Heidelberg.

[5] Sitthisak, O. 2009. A Competency Model for Semiautomatic Question Generation in Adaptive Assessment. Ph.D. thesis, Engineering, Science and Mathematics Faculty, University of Southampton.

[6] Papanikolaou, K. A. and Grigoriadou, M. 2003. An Instructional Framework Supporting Personalized Learning on the Web. In Advanced learning technologies. The 3rd IEEE international conference on (pp. 120-124). IEEE.

[7] Gouli, E., Kornilakis, H., Papanikolaou, K. and Grigoriadou, M. 2001. Adaptive Assessment Improving Interaction in an Educational Hypermedia System. In Proceedings of the PanHellenic Conference with International Participation in Human-Computer Interaction.

[8] I. Lmati et al., 2015.Alignment between two domain ontologies (Case of educational orientation in mathematics education). 5th International Conference on Information \& Communication Technology and Accessibility (ICTA). Marrakech, pp. 1-3.

[9] I. Lmati, E. Benlahmar, N. Achtaich, 2015. Enrichment and population of an educational ontology from a corpus of mathematical analysis. Journal of Theoretical and Applied Information Technology, vol. 82.

[10] I. Lmati, E. Benlahmar, N. Achtaich, 2014. A new approach for generating exercises based ontologies: A case study of courses in mathematical analysis. Journal of Theoretical and Applied Information Technology, vol. 70 , no 3 .

[11] F. Kamareddine, M. Maarek, K. Retel, et al. 2007. Narrative structure of mathematical texts. In : Towards Mechanized Mathematical Assistants. Springer Berlin Heidelberg. p. 296-312.

[12] A. Bouzeghoub, B.Defude, J.F. Duitama, C. Lecocq, 2005. A model for semantic description of educational resources based on a domain ontology. Science and Technologies of Information and Communication for Education and Training, Volume 12. 